\title{
REFLEXIONES Y PROPUESTAS DE SOLUCIÓN SOBRE LA CRISIS AMBIENTAL: EL CASO DE LA EncruciJada, Chiapas
}

\section{Juan Toledo Medina ${ }^{1}$}

\section{Resumen}

El presente artículo tiene el propósito de esbozar la crisis ambiental a nivel mundial, la degradación de la biodiversidad en el territorio mexicano, y la degradación de la reserva de la Encrucijada Chipas. Para los dos primeros niveles, se realizó una revisión documental, con el objeto de explicar las causas de ésta, que se manifiesta en la pérdida de bosques y selvas, el calentamiento global, la contaminación del aire, suelo, ríos, mares y lagos. Los primeros intentos de solucionar esta crisis ambiental a nivel mundial los llevó a cabo la ONU, al organizar las cumbres internacionales de 1972, 1992, 1997 y 2002. Aunque en este mismo periodo, también surge el modelo de desarrollo sustentable, la Economía Ambiental y la Economía Ecológica, sin embargo, lamentablemente la degradación de los ecosistemas sigue creciendo. En cuanto a la Encrucijada, el problema se abordó documental y empíricamente, obteniéndose como resultado que las causas que degradan los manglares, humedales y contaminan los cuerpos acuíferos, matan aves, mamíferos y peces, así como la generación de enfermedades en algunas personas que viven en el litoral; son la falta de sustentabilidad de las actividades agrícolas, ganaderas, mineras e industrial, los incendios, el azolvamiento de los esteros, la poca eficiencia de las políticas ambientales, y la falta de consciencia ambiental de los actores sociales y políticos.

Palabras clave: Crisis ambiental, sustentabilidad, políticas ambientales, degradación de ecosistemas.

1 Mtro. por la Universidad Autónoma de Chiapas, Profesor de tiempo completo en la Facultad de Ciencias Sociales de la UNACH. Perfil PRODEP deseable. Correo: jdtmedina@gmail.com 


\section{Abstract}

This article is intended to outline the environmental crisis worldwide, the degradation of biodiversity in Mexican territory, and the degradation of the crossroads sparks reserve. For the first two levels, we performed a documentary review, in order to explain the causes of this, which manifests itself in the loss of forests, global warming, and pollution of air, soil, rivers, seas and lakes. The first attempts to try to solve this environmental crisis worldwide, conducted UN organizing international summits from 1972, 1992, 1997 and 2002. Although during this period, also the model of sustainable development, environmental economics and ecological economics, however unfortunately the degradation of ecosystems continues to grow. As for the crossroads, the problem addressed documentary and empirically, resulting in that the causes that they degrade the mangroves, wetlands and pollute water bodies, killing birds, mammals and fish, as well as the generation of diseases in Some people living on the coast; they are the lack of sustainability of activities agricultural, livestock, mining and industrial, fire, the siltation of the estuaries, the low efficiency of environmental policies, and the lack of environmental awareness of the social and political actors.

Keywords: sustainability, environmental Crisis, degradation of ecosystems, environmental policies.

\section{Introducción}

El presente artículo tiene el propósito de analizar el problema de la degradación ecológica en el mundo, en México y en la reserva de la Encrucijada. Tomando como elementos de análisis las zonas boscosas, las características de las actividades económicas, las políticas ambientales y la cultura ambiental de la población humana.

A nivel mundial el problema ambiental tiene sus manifestaciones en la destrucción de la capa de ozono, la contaminación del suelo, el aire, ríos, lagos, mares y océanos; la pérdida de superficies selváticas, de bosques, y el calentamiento global por el efecto invernadero, producto del exceso de bióxido de carbono generado por el consumo de combus- 
tible fósil. Lo anterior tiene su origen en la poca sustentabilidad de las actividades económicas, la falta de conciencia ambiental, la naturaleza de las políticas ambientales y la expansión de las zonas urbanas.

Para algunos ambientalistas, la crisis ambiental es resultado de la crisis de la civilización, es un problema de pensamiento humano que se torna más intensa en las regiones subdesarrolladas, en las que predomina el interés por la rentabilidad económica, sacrificando la racionalidad ambiental.

En la década de los sesentas y setentas, ante la crisis ambiental se cuestionó el modelo de desarrollo de tipo occidental prevaleciente, debido a que este modelo había generado el agotamiento de muchos recursos naturales y por consiguiente la alteración de los ecosistemas.

Ante tal panorama las primeras acciones para disminuir los problemas ambientales en el mundo, fueron realizadas por parte de la ONU, que organizó las cumbres internacionales de 1972 en Estocolmo, Suecia; en Río de Janeiro, Brasil, en 1992; y en Johannesburgo, Sudáfrica, en 2002. De estas cumbres surgieron propuestas de acción, para que los gobiernos de las naciones que participaron en éstas las pusieran en práctica con el objetivo de incidir en la preservación de la biodiversidad.

En la primera cumbre se propuso el modelo de Ecodesarrollo, y se retoma el principio de que todos tenemos derecho a vivir en un ambiente sano. Para la década de los ochentas del siglo XX, todas las teorías económicas y sus estrategias de desarrollo entraron en crisis y como alternativa se propusieron nuevos modelos de desarrollo bajo el principio de sustentabilidad, que popularizó el Informe Brundtland en 1987.

Asimismo, surge la implementación de un programa de Educación Ambiental, formal y no formal, por parte de la ONU y la UNESCO. Sin embargo estas propuestas lamentablemente no han logrado los frutos que se esperaban, ya que la cultura ambiental no se ha modificado, y los problemas ambientales siguen incrementando.

También se propuso como medida de solución la implementación de la economía ambiental, que deriva de la economía tradicional o neoclá- 
sica, pero ésta ha presentado limitaciones tanto epistemológicas como de aplicación a la solución de los problemas ambientales, por lo que, como una nueva alternativa, surge el modelo de la Economía Ecológica o Economía Verde. El cual se erige fuera de los cánones de la economía tradicional y plantea realizar actividades económicas sustentables, pero hasta ahora su influencia es ínfima.

En el territorio mexicano, el problema ambiental se analiza específicamente sobre las áreas naturales protegidas, que representa el 1.6\% del territorio nacional. En ellas no se observa una adecuada conservación, debido a un mal funcionamiento administrativo de las instituciones que se encargan de ésta, así como por la confluencia de diversos factores. Como los siguientes: los asentamientos humanos en estas zonas, que poco a poco van cambiando el uso del suelo, lo que reduce la extensión de las áreas protegidas; las actividades económicas como la agricultura, la ganadería, la pesca, el comercio, la cacería, los desmontes, la explotación clandestina de los bosques, la contaminación del agua; la falta de una adecuada normatividad de conservación entre otros aspectos. A pesar de la implementación de las políticas ambientales por parte de las instituciones oficiales representadas por la CONABIO, la SEMARNAT y la CONANP.

En la problemática ambiental se incluye el estudio de la reserva biosfera de la Encrucijada, en el litoral del estado de Chiapas, que se localiza en las Regiones Istmo- Costa y el Soconusco, decretada reserva el 6 de junio de 1995, con una extensión de 144,868 hectáreas. Presenta una creciente degradación, sobre todo en la devastación de zonas de manglares en la Región del Soconusco, como causas de la expansión urbana, el impacto negativo del desarrollo de actividades agrícolas, ganaderas, la minera, la industria azucarera, los incendios, la explotación de la madera de mangle y los asentamiento humanos. Además de la poca eficiencia de las políticas ambientales implementadas hasta ahora.

La investigación en la Encrucijada tiene como objetivo conocer los diversos factores que inciden en la degradación de los bosques de mangles, humedales, la contaminación del recurso acuífero y la muerte de alguna parte de la fauna por la influencia de sustancias tóxicas. 
Para obtener la información, se realizó una revisión documental y la investigación empírica, identificándose como unidades de análisis: los bosques de manglares de la zona núcleo, y de la zona de amortiguamiento, las diversas actividades económicas, las políticas ambientales, las cooperativas, los niveles de conciencia ambiental de las comunidades, y las instituciones ambientalistas que aquí participan.

\section{Los problemas ambientales en el mundo y las alternativas de solución}

Existen múltiples reacciones y protestas por parte de grupos ambientalistas, que forman parte de la sociedad civil y de las ONG, en distintos espacios del planeta Tierra, debido a la angustiante crisis ambiental actual, para la que hasta ahora no ha habido respuestas contundentes de solución, solo se han implementado algunos paliativos, por parte de los actores económicos, sociales y políticos de las naciones, sobre todo en los países subdesarrollos.

Buena parte de la literatura establece que los actores directamente responsables del origen del problema ambiental son los empresarios y algunos gobiernos de naciones, en la mayoría de las Regiones subdesarrolladas; los que no han logrado comprender que en esta tendencia, también está en peligro la existencia del ser humano.

Como consecuencia de los desequilibrios ecológicos y del calentamiento global de la tierra, la humanidad se enfrenta a la falta de alimentos por la carencia de recursos naturales, la escasez de agua potable y la vulnerabilidad a padecer diversas enfermedades, producto de la contaminación del suelo, del aire y de las aguas de los ríos, lagos, mares y océanos, así como la destrucción de la capa de ozono.

El problema ambiental, es el resultado de la forma en que la sociedad humana se ha relacionado con su entorno natural, en la que el desarrollo tecnológico no ha sido acorde con la conservación de la naturaleza y no ha permitido la armonía de la sociedad humana con el medio ambiente natural. Se vive en un esquema económico en el que predomina la filosofía antropocéntrica, que no toma en cuenta la regeneración de los 
recursos naturales y tampoco da importancia al bienestar de las generaciones presente y futuras.

Es absurdo que "la racionalidad de la humanidad" genere en la actualidad una irracionalidad ambiental, lo que ha ocasionado el caos de los ecosistemas. Por lo que algunos intelectuales proponen el crecimiento económico cero, al suponer que la causa del problema es el crecimiento económico. Para Leff (2008) la crisis ambiental es una crisis civilizatoria que se manifiesta en la presencia de externalidades negativas, ya que la economía tradicional o ambiental, que deriva de la economía neoclásica, privilegia la rentabilidad de las actividades económicas, sometiendo todo a la lógica del mercado, incluyendo a los bienes públicos como el medio ambiente.

Por su parte, Martínez (2010) menciona que la crisis ambiental actual es el resultado de la forma como el hombre se ha relacionado con la naturaleza, es un problema de responsabilidad ante la vida, ante la historia y ante sí mismo. Por consiguiente, la transformación de la naturaleza al utilizar sus recursos, tiene un carácter y un interés privado, pero la contrapartida es que se pone en peligro la biodiversidad y la reproducción de la vida humana.

Leff (2008) establece que en este contexto los territorios más vulnerables, en los próximos 50 años, son las zonas intertropicales en las que se encuentran los países subdesarrollados y en ellos las poblaciones indígenas. La práctica económica del régimen actual ha generado desequilibrios en los sistemas ecológicos, el agotado de los recursos naturales y el calentamiento global de la Tierra, a través del efecto invernadero causado principalmente por el uso de energías fósiles, gases y compuestos químicos de origen industrial.

Los países emisores de la mayor cantidad de gases de efecto invernadero en el mundo son Estados Unidos y China. Aunque este problema ambiental se podría contrarrestar con la presencia de una mayor masa forestal, lamentablemente está ocurriendo lo contrario; el plantea presenta una impresionante deforestación (Leff, 2008) que, nadie puede o quiere detener, se atiza como resultado de la expansión urbana, la ex- 
plotación de madera para fines comerciales, y de la explotación de los recursos mineros en los espacios boscosos y selváticos.

El problema ambiental requiere de una inmediata y urgente solución, ya que no se puede continuar con la destrucción de la tierra, que sufre el creciente calentamiento global, la contaminación atmosférica, la destrucción de la capa de ozono, la pérdida de bosques y selvas tropicales, y con ello la muerte de la fauna. La presencia de toneladas de basura, la contaminación con aguas residuales, por residuos nucleares, agroquímicos y sustancias venenosas derivadas de la industria y la minería, que contaminan los suelos ríos, mares y lagos; además de la disminución y escasez del agua dulce para el consumo humano.

Según Martínez (2010) la crisis ecológica actual es provocada por la voracidad del sector privado (nacional y transnacional) y público, sobre los recursos naturales como el agua, el suelo, la tierra, los bosques, los minerales y las bellezas escénicas. Se trata de un modelo de vida occidental, un modelo de desarrollo dominante que da prioridad a una economía insustentable e injusta desde el punto de vista social.

Hasta ahora el sector público se ha mostrado incapaz de detener la destrucción de la biodiversidad. Ante la práctica económica empresarial del sistema prevaleciente, la cual fomenta e impulsa la explotación de los recursos naturales con la finalidad de obtener rentabilidad sin considerar la conservar de los ecosistemas (Martínez, 2010), lo único que hasta ahora ha hecho el sector público es crear políticas ambientales, que en esencia solo han paleado el problema.

Los antecedentes de una acelerada depredación irracional de la tierra se remonta a la primera Revolución Industrial del siglo XVIII, en favor de un modelo de crecimiento sostenido no sustentable que ha atentado contra la naturaleza. No es sino hasta la década de los cincuenta del siglo pasado, cuando los investigadores comenzaron a preocuparse por los problemas ambientales. (Según Guzmán, San Martín y Barber, 2010; citado por Martínez 2010) 
En la década de los sesentas y setentas, surgió la incertidumbre de mantener o no el modelo de desarrollo occidental, (Toro, 2007) ya que no mejora las condiciones de vida de la población, además de generar la degradación de los ecosistemas.

Los organismos oficiales y equipos de investigación de prestigio, advirtieron que las tendencias de crecimiento de la economía y de la población mundial podrían provocar un colapso ambiental en un futuro cercano. Se mencionó que el modelo de desarrollo económico generaba el agotamiento irreversible de los recursos naturales como las fuentes de energía, minerales, recursos forestales, pesqueros, suelo, agua; la reducción de la capa de ozono y la pérdida de la biodiversidad (Toro, 2007).

El calentamiento global de la Tierra está creciendo de manera vertiginosa, en la década de los noventa, y específicamente el año de 1998, la tierra estuvo más caliente desde 1861 , justo en el siglo XX hubo más calentamiento global. Dado esta tendencia se prevé que la temperatura promedio global de la Tierra se incrementará de 1.4 a 5.8 grados centígrados entre 1990 y 2100 , mientras que en 1861 el incremento solo fue de 0.6 grados centígrados (Leff, 2008).

Como se mencionó, la primera organización internacional que impulsó el análisis y reflexión de los problemas ambientales fue la ONU. La cumbre internacional en Estocolmo, Suecia (1972), evaluó los daños ocasionados a la naturaleza y tomó medidas para sanearla. Los países que participaron en esta cumbre retomaron el derecho que todos tenemos a un ambiente sano, por lo que se adoptó el modelo ecodesarrollo (Gutiérrez, 1996; Contreras, 2002).

Para la década de los ochentas, todas las teorías económicas y sus estrategias de desarrollo entraron en crisis, manifestándose en los aspectos económicos, sociales y ambientales, sobre todo en los países subdesarrollados, especialmente en África y América Latina (Gutiérrez, 1996). Por lo cual se plantearon nuevos modelos de desarrollo como el desarrollo humano: es un concepto multidimensional y crítico que incorpora aspectos ideológicos y éticos, con el propósito de hacer sos- 
tenible el desarrollo económico y social, contrariamente a la filosofía antropocéntrica (Toro, 2007).

El término de sustentabilidad apareció por primera vez en 1974 aplicado por Henry Kissinger, jefe de la diplomacia estadounidense, quien lo propuso en la declaración de Cocoyoc, Morelos, como un subtítulo del Ecodesarrollo ${ }^{2}$. Siendo en 1980 cuando la Unión Internacional para la Conservación de la Naturaleza y sus Recursos utilizó la sustentabilidad en la elaboración de una estrategia mundial para la conservación y no para el desarrollo. Pero el término se hizo popular en 1987 por medio del Informe de Gro Harlein Brundtland, primera ministra de Noruega, titulado "Futuro Común", que fue publicado en 1988 a cargo de la Comisión Mundial sobre Medio Ambiente y Desarrollo, como propuesta de la ONU. El modelo aplicó el principio de equilibrio entre la economía, la ecología y la sociedad (Gutiérrez, 1996).

Para seguir abonando a la solución de los problemas ambientales la ONU celebró en 1992 "la Cumbre de la Tierra", en la que se reconoció que la crisis ambiental representa el agotamiento de un estilo de desarrollo que depreda la ecología, por lo que requiere de cambios profundos en el modelo actual (Gutiérrez, 1996).

De acuerdo con Landa (2010), la degradación del medio ambiente es atizada por la fuerte deforestación mundial, que asciende a 13 millones de hectáreas. En la última década, desaparecieron sistemas forestales completos en 25 países y en 29 países más disminuyeron en $90 \%$, perdiéndose cada año 7.8 millones de hectáreas de bosques por cultivos de subsistencia en laderas y por pérdidas del rendimiento en tierras degradadas.

2 El programa de las Naciones Unidas para el Medio Ambiente (PNUMA) y la Confederación de las Naciones Unidas para el Comercio y el Desarrollo (UNCTAD), invitaron a destacadas personalidades y especialistas en el campo del desarrollo, así como a expertos sobre problemas del medio ambiente, a participar en el Simposio sobre "Modelos de utilización de recursos, medio ambiente y estrategias de desarrollo", auspiciado por el gobierno de México y realizado en Cocoyoc, Morelos, del 8 al 12 de octubre de 1974 (Revista Comercio exterior, 1975). 
Otra medida de solución al problema ambiental, fue la implementación de un programa de educación ambiental, tanto formal como no formal, que se derivó de la cumbre de Naciones Unidas para el medio Ambiente, (PNUMA) en 1972 a través de la cooperación de la UNESCO y el PNUMA.

Pero antes de este programa, existieron otros intentos para implementar una educación ambiental en Europa por parte de la ONU, en Lucerna, Suiza (1966), donde se realizó el Simposio sobre educación en materia de conservación, para 1969 esta iniciativa se realizó en Estados Unidos (Contreras, 2002). Con el mismo sentido, se creó en Suecia un organismo que se encargaría de controlar e inspeccionar la educación ambiental a nivel escolar y en la decimosexta sesión de la conferencia de la UNESCO, el 30 de octubre de 1970, se creó el Programa MAB (el hombre y la Biósfera) (Gutiérrez, 1996).

En 1992 la ONU realizó en Río de Janeiro la reunión denominada "Cumbre para la Tierra" en la que participaron 179 países, destacando la incorporación del concepto de desarrollo sostenible como eje para las diversas acciones gubernamentales y de los organismos internacionales. En esta reunión se integró la agenda 21, con un conjunto de normas para transitar hacia un desarrollo sostenible y una declaración de principios en materia de bosques. De manera paralela, la mayoría de los gobiernos, con la ausencia de algunos países desarrollados, particularmente de los Estados Unidos de América, suscribieron la Convención sobre el Cambio Climático, con énfasis en el control de los gases que provocan el efecto invernadero (Leff, 2008).

De acuerdo con Leff (2005) esta cumbre marca un punto de inflexión en la geopolítica ambiental global ya que a partir de aquí las políticas del desarrollo sostenible han puesto en operación un nuevo marco legal internacional; basado en acuerdos multilaterales que incluyen instrumentos jurídicos para establecer normas a los agentes económicos y sociales, que limiten y reviertan el impacto de los procesos económicos y tecnológicos sobre el medio ambiente. 
De los convenios bilaterales, los más controvertidos han sido el cambio climático y la diversidad biológica, por sus implicaciones globales y la variedad de intereses y conflictos, poniendo de manifiesto las dificultades para internalizar los costos ecológicos y conciliar las políticas económicas y ambientales.

Las dificultades para poner en práctica los acuerdos multilaterales manifiestan las resistencias del orden económico de internalizar los costos ambientales y acomodarse a las normas de sustentabilidad. Un ejemplo de esto son los obstáculos interpuestos para el cumplimiento de los acuerdos de Río de Janeiro en 1992, con el objetivo de limitar las emisiones de gases de efecto invernadero y frenar el avance del calentamiento global del planeta (Leff, 2005).

Pero debido a que no se obtuvieron los resultados esperados, se organizó el protocolo de Kioto, Japón en 1997, para reforzar los esfuerzos de los países en la reducción de dichos gases, pero tampoco se consiguen los resultados deseados, por lo que se vuelve a realizar otra Cumbre sobre el cambio Climático en París, Francia en 2015.

En el 2002 tuvo lugar la cumbre mundial de Johannesburgo que versó sobre el desarrollo sustentable, aquí se adoptaron los objetivos del Desarrollo del Milenio, sobre el combate al calentamiento global, el cambio climático, mejorar la educación, resolver el problema de pobreza, proveer agua limpia a la población, el saneamiento y la renovación de la energía hacia energía limpia, con el objeto de garantizar la sobrevivencia de la humanidad y del planeta. Pero todo esto dependió de las políticas públicas implementadas, y del cambio de racionalidad en la economía (Leff, 2008).

Según Toro (2007) el proyecto de desarrollo sostenible, debe conciliar dos dimensiones confrontadas, que son el progreso humano y la conservación de los recursos naturales. El propósito del desarrollo sostenible debe ser de carácter global pero intercalado con lo local. En este sentido, la geografía propone una concepción holística, integradora y sistemática de las relaciones entre la humanidad y el medio ambiente. 
La geografía tiene una perspectiva territorial de la sostenibilidad, siendo el territorio que tiene un carácter escalar donde acontecen los fenómenos económicos, sociales, políticos, ecológicos y éticos.

Para contribuir al fortalecimiento de un modelo de desarrollo sustentable, en cualquier territorio, se requiere del apoyo de las políticas públicas de los gobiernos nacionales y locales, y de la sinergia de los distintos actores sociales, bajo un proyecto de desarrollo ambiental.

Para Torres y Santander (2013), las políticas públicas son el reflejo de los ideales de la sociedad y expresan los objetivos del bienestar colectivo, que permiten orientar el desarrollo y cómo hacerlo entre los distintos actores sociales. Las políticas públicas son la materialización de la acción del Estado y la ciudadanía. Éstas legitiman al Estado y tienen un carácter instrumental para proveer los servicios básicos o la administración de los bienes públicos.

De acuerdo con Roth (2002) las políticas públicas son programas de acción que representan la realización de decisiones colectivas, y son un medio usado por el Estado en su voluntad de modificar comportamientos mediante el cambio de las reglas operantes hasta un momento determinado. En relación a la administración de los bienes comunes, Nowlin (2011) plantea que las políticas públicas se entienden como los arreglos institucionales que permiten o prohíben acciones.

Por su parte Ostrom (2007) considera que pueden existir arreglos institucionales distintos al mercado o al Estado para resolver problemas de acción colectiva, entre los que se incluyen el nivel local y las organizaciones de autogobierno. Una extensión del modelo propuesto por Ostrom introduce los sistemas sociales y ecológicos. Establece que el análisis institucional y del desarrollo, tiene un carácter de multinivel, donde existen espacios sociales en que los individuos interactúan e intercambian bienes y servicios, y también resuelven sus problemas y se dominan unos a otros.

En las políticas públicas es importante mencionar la gobernanza, que se entiende como la capacidad de realizar una política pública en 
colaboración con el gobierno y los actores sociales y políticos, (Hufty, 2008). Aquí el gobierno requiere del acompañamiento y la cooperación de múltiples actores para, ya no como único objetivo de ejecutar lo planeado, garantizar la coproducción y cooperación de los actores claves (Börzel, 1997).

La propuesta más contundente en el campo de la Ciencia Económica para la solución de los problemas ambientales es la Economía Ecológica, después del cuestionamiento de la Economía Ambiental, que deriva de la Economía tradicional y que no garantiza la sustentabilidad. Otra propuesta viable es, la puesta en marcha de una educación ambiental heterodoxa, que realmente transforme la cultura ambiental en favor de la conservación de los ecosistemas.

\section{Las políticas ambientales y la conservación de la biodiversidad en México}

De acuerdo con la Comisión Nacional de Áreas Naturales Protegidas (2014) las políticas de conservación de la biodiversidad en México inicia en 1876, con la protección del Desierto de los Leones que tiene el propósito fundamental de asegurar la conservación de 14 manantiales que abastecían de agua a la Ciudad de México, a principios del siglo XX se decreta como el primer Parque Nacional de la República Mexicana.

Paradójicamente, en la década de los setentas esta política de protección de las áreas naturales protegidas entró en contradicción por la tendencia del poblamiento de las zonas selváticas y boscosas, con el objetivo de descongestionar las ciudades, destruyendo los ecosistemas con los procesos de ganaderización y la actividad agrícola, los que fueron subsidiados por el propio gobierno mexicano (CONANP, 2014).

Las políticas de conservación de la biosfera tuvieron su auge en el periodo del cardenismo (1935-1940), en el que se crearon la mayoría de los parques nacionales de México, siendo en 1996 un total de 36, distribuidos en 17 entidades de la República Mexicana, con una superficie total de 800,000 hectáreas. Aunque para Sosa y Quadri de la Torre, los parques nacionales son 40 (Gutiérrez, 1996). 
Pero en la década de los ochenta y noventa, los esfuerzos de la sociedad civil y la participación de los grupos académicos de las principales instituciones de educación superior del país, propiciaron que se reactivaran las políticas de protección y la creación de las áreas nacionales protegidas (CONANP, 2014).

Según Gutiérrez (1996) algunos especialistas de la problemática ambiental de México, coinciden en que las áreas naturales protegidas no presentan un buen funcionamiento administrativo que faciliten el cumplimiento de los objetivos ambientales. Además, los parques nacionales han reducido su extensión debido a los cambios del uso del suelo. Para 1996 en México solo existe el 1.6\% del territorio nacional cubierto de parques, reservas forestales y refugios de faunas silvestres. Este porcentaje se encuentra muy por debajo de otros países del continente americano.

El 69\% de los parques nacionales tienen asentamientos humanos y son degradados por las actividades ganaderas, agrícolas, comerciales, pesqueras, la cacería, los desmontes, la explotación clandestina de los bosques maderables, los bancos de materiales, la erosión ocasionada por motociclistas y caminantes, la contaminación del agua, los saqueos de tierra, el saqueo de los recursos forestales autorizado por las autoridades y el saqueo de restos arqueológico.

Algunos espacios se han convertido en basureros que contaminan la muerte de árboles, entre otras causas que provocan su degradación; sin que exista una adecuada normatividad que garantice su conservación (Gutiérrez (1996), lo que demuestra una falta de conciencia ambiental y de sustentabilidad de los actores sociales y políticos en la sociedad mexicana.

Como parte de las políticas ambientales de México se creó en 1992 la Comisión Nacional para el Conocimiento y Uso de la Biodiversidad (CONABIO) que se conforma por representantes de nueve Secretarías de Estado, siendo su presidente el ejecutivo de la República Mexicana y el secretario técnico de la Secretaría del Medio Ambiente y Recursos Naturales (SEMARNAT). Se suman a la comisión el titular de la Secretaría 
de Agricultura Ganadería Desarrollo Rural Pesca y Alimentación (SAGARPA) y los titulares de la Secretaría de Desarrollo Social (SEDESOL): Secretaría de Economía (SE), Secretaría de Salud (SSA), Secretaría de Turismo (SECTUR), Secretaría de Educación Pública (SEP), Secretaría de Energía (SENER) y Secretaría de Hacienda y Crédito Público (SHCP). Poco después se creó el Fondo Mexicano para la Conservación de la Naturaleza, bajo la aportación financiera del sector privado y grupos filantrópicos (CONANP, 2014).

La CONABIO tiene el objetivo principal de preservar el capital natural, ésta sirve de puente entre la academia, el gobierno y la sociedad. Sus funciones más importantes consisten en instrumentar y operar el sistema nacional de información sobre la biodiversidad, informar y asesorar a diversos usuarios, así como instrumentar redes de información nacional y mundial sobre la biodiversidad (CONANP, 2012).

Por su parte, la Secretaría del Medio Ambiente y Recursos Naturales, es la encargada de hacer cumplir la legislación ambiental, para lo cual se auxilia del Instituto Nacional de Ecología, La Procuraduría Federal de Protección al Medio Ambiente y la Comisión Nacional del Agua (Borrego, 2006).

La primera vez que se implementaron las políticas de protección del medio ambiente, de manera institucional y como una obligación del Estado, fue en 1982, con la implementación de la Ley de Protección del Ambiente, la que sufrió algunas modificaciones menores en 1988, sin alterar su estructura original. Dos años después, se creó la Secretaría de Desarrollo Urbano y Ecología, con la finalidad de vigilar la aplicación de la Ley, sin embargo, sus funciones se centraron en la prevención y control de la contaminación (Borrego, 2006).

Con el Gobierno de Ernesto Zedillo Ponce de León, en 1994 se creó la Secretaría del Medio Ambiente Recursos Naturales y Pesca (SEMARNAP) para proteger y restaurar los ecosistemas y recursos naturales. En sus lineamientos se estableció el vínculo entre las políticas ambientales y el combate a la pobreza, que tiene su origen en el compromiso contraído por el país con la firma de la agenda del siglo XXI, derivada 
de la Conferencia de Río de Janeiro de 1992, con la pretensión de lograr el desarrollo sustentable (Borrego, 2006). Dentro de este modelo se crearon los Consejos Consultivos de desarrollo sustentable, uno a nivel nacional y cuatro a nivel regional, pero tales políticas ambientales no lograron avances importantes en la preservación de la biodiversidad.

Para 1996, el gobierno federal realizó la reforma de la Ley General de Equilibrio Ecológico y Protección al Medio Ambiente (LGEEP) y en 2000 se conformó la Comisión Nacional de Áreas Naturales Protegidas (CONANP), como órgano descentralizado de la Secretaría del Medio Ambiente y Recursos Naturales.

La CONANP tiene la función de conservar las áreas nacionales protegidas y establece que para alcanzar la sustentabilidad se debe involucrar a la población, lo cual plantea la conformación de una cultura que permita la conservar y valorar la biodiversidad y los ecosistemas. Esta cultura se forja con la educación formal, la no formal y la informal, la capacitación, la participación y el uso de la comunicación estratégica. Además, desarrolla sus funciones bajo los programas de conservación para el desarrollo sustentable de empleo temporal, de vigilancia comunitaria en áreas naturales protegidas, de monitoreo biológico en áreas naturales protegidas, el programa para la integración o modificación de los programas de manejo de áreas naturales protegidas, entre otros aspectos (Borrego, 2006).

De acuerdo con la CONANP (2012), es necesario contar con los conocimientos bilógico y ecológico si se quieren alcanzar los objetivos de conservación de las áreas protegidas, que deben integrarse a las actividades económicas y a la sociedad.

México es uno de los países con mayor capacidad de sistemas ecológicos y biodiversidad en su territorio, para ello la CONABIO ha participado en la ejecución de programas de cooperación en el marco del comité conjunto México-EE.UU., sobre la conservación de la vida silvestre, y en el comité tripartita México-Canadá-EE.UU., en el desarrollo de las estrategias de conservación (SEMARNAT, 2012). 
Las áreas naturales protegidas son un instrumento de política gubernamental que pretende la protección de la biodiversidad y promueven la importancia ecológica, social y económica de estas áreas, como sitios propicios para el crecimiento regional sustentable, en beneficio de la población que habita dentro de ellas o en zonas de influencia.

A pesar de los esfuerzos lamentablemente México ha tenido una pérdida importante de su capital natural, ya que ha desaparecido el 95\% de sus bosques tropicales. Según datos de SEMARNAT la deforestación avanzó en los noventa a un ritmo de 348,000 hectáreas anuales y 75\% del territorio presenta diferentes grados de erosión relacionados con la pérdida de biodiversidad (Leff, 2000), mientras que para Galicia (2013) la pérdida anual de zonas boscosas asciende a 40 mil hectáreas anuales.

México estuvo cumpliendo formalmente, en su momento, con sus compromisos de la agenda ambiental global y comprometiéndose con el cumplimiento de los objetivos de Desarrollo del Milenio, sin embargo, las políticas públicas nacionales no han alcanzado la escala de acuerdos y acciones que requiere el país para lograr un desarrollo sustentable (Leff, 2000).

Si México ha avanzado en la creación de sus instituciones ambiental, como la CONABIO, la SEMARNAT, la CONANP, la Comisión Nacional del Agua, la procuraduría del Ambiente, el Ordenamiento Territorial, el Sistema Nacional de Áreas Protegidas, así como la implementación de políticas ambientales de sustentabilidad en la economía y el cambio hacia una cultura sustentable, lamentablemente, a pesar de estos esfuerzos, no se ha logrado detener la degradación de los ecosistemas.

La sustentabilidad en México se presenta como un gran desafío para las políticas públicas, y una enorme responsabilidad en los diferentes niveles de gobierno, así como en los órganos legislativos, del sector empresarial, la comunidad científica (Leff, 2000) y la sociedad civil. 


\section{Los niveles de sustentabilidad en la reserva biosfera de la Encrucijada, Chiapas}

El estado de Chiapas cuenta con un litoral de $270 \mathrm{~km}$, en el que se localizan esteros y lagunas, que tienen una superficie de 75, 828 hectáreas, subdivididas en tres subsistemas que son de Mar Muerto: La Joya Buena Vista, Carretas Pereyra y Chantuto-Panzacola.

En el último se localiza la reserva de La Encrucijada, conformada por manglares y humedales, siendo éstos de los más importantes a nivel nacional e internacional, por lo que la Encrucijada fue declarada sitio RAMSAR el 20 de marzo de 1996 (RAMSAR) ${ }^{3}$.

La reserva de La Encrucijada tiene una extensión de 144, 868 hectáreas, que representa el $1.67 \%$ de la superficie total de 28 áreas piloto definidas en el Programa Nacional de Áreas Naturales Protegidas de 19952000 (SEMARNAT y La Comisión Nacional de Acuacultura y Pesca, 2009), decretándose reserva de la biosfera desde el 6 de junio de 1995.

La reserva está comprendida en seis municipios que son: Mazatán, Acapetahua, Huixtla, Villa Comaltitlán, Mapastepec y Pijijiapan. Así mismo pertenece a dos regiones económicas la X Soconusco, donde se encuentran los municipios de Acapetahua, Huixtla, Mazatán y Villa Comaltitlán; y la región IX Istmo-Costa, en la que están los municipios de Pijijiapan y Mapastepec (CONANP, 2013).

La Encrucijada se conforma por una zona núcleo de 36,216 hectáreas y una zona de amortiguamiento, con extensión de 108, 651 hectáreas, siendo esta última la que mayor deterioro ha sufrido. En la zona núcleo se ubica la comunidad de El Castaño, la isla y comunidad de La Concepción.

3 Convenio RAMSAR, realizado en la ciudad con el mismo nombre en Irán en 1971, pero entra en vigor en 1975. Tiene como objetivo conservar los humedales. México se suma al convenio en 1986, por la CONANP que la encargada de aplicar los convenios. México cuenta con 142 sitios RAMSAR, con una superficie de casi nueve millones de hectáreas, que incluyen recursos como humedales, manglares, arrecifes de coral, oasis, sistemas cársticos y especies amenazadas (SEMARNAT, 2013). 
La Encrucijada comenzó a protegerse y conservarse desde 1954, como parte de la política pública ambiental a nivel estatal, desde las visitas del ambientalista Dr. Miguel Álvarez del Toro dicha reserva ya era conservada por el Instituto Natural del Gobierno de Chiapas (CONANP, 2014). Para 1972 La Encrucijada pasó a ser patrimonio del estado de Chiapas, a través de un decreto del gobierno del estado de Chiapas, declarándose Área Natural y Típica del estado de Chiapas, tipo ecológica manglar Zapotón, con una extensión de 2,500 hectáreas.

En la década de los ochentas del siglo pasado, y de acuerdo con la legislación federal, la reserva fue administrada por la Secretaría de Desarrollo Urbano y Ecología (SEDUE), la que después amplió su extensión a 36,000 hectáreas; en 1988 nuevamente el Instituto de Historia Natural del gobierno del estado de Chiapas, se hizo cargo de ella (CONANP (2014). Sin embargo, de acuerdo con la CONANP (2014), los trabajos de conservación sistemático y formal de la reserva iniciaron en 1991, a través del Instituto de Historia Natural (IHN), consolidándose con el decreto presidencial del seis de junio de 1995, como reserva de la biosfera, respetándose la pequeña propiedad o propiedad social, y evitando las expropiaciones de territorios a la población residente en la reserva.

Para desarrollar un adecuado trabajo de seguimiento en la conservación de la biodiversidad, se conformó un consejo de asesores, integrado por la Universidad Autónoma de Chiapas (UNACH), la Universidad de Ciencias y Artes de Chiapas (UNICACH), ECOSUR, los Ayuntamientos Municipales, la Federación de Cooperativas Pesqueras, algunas Organizaciones no Gubernamentales, representantes de comunidades, grupos de prestadores de servicios turísticos y de productores acuícolas, grupos organizados de productores agrícolas, ganaderos y diversos grupos comunitarios organizados (CONANP, 2014).

De acuerdo con la CONANP (2014), la población humana que vive en el interior de la reserva, está integrada por 73 comunidades, en las que residen un total de 79 mil personas. 


\section{Mapa 1. Delimitación de la reserva de La Encrucijada}

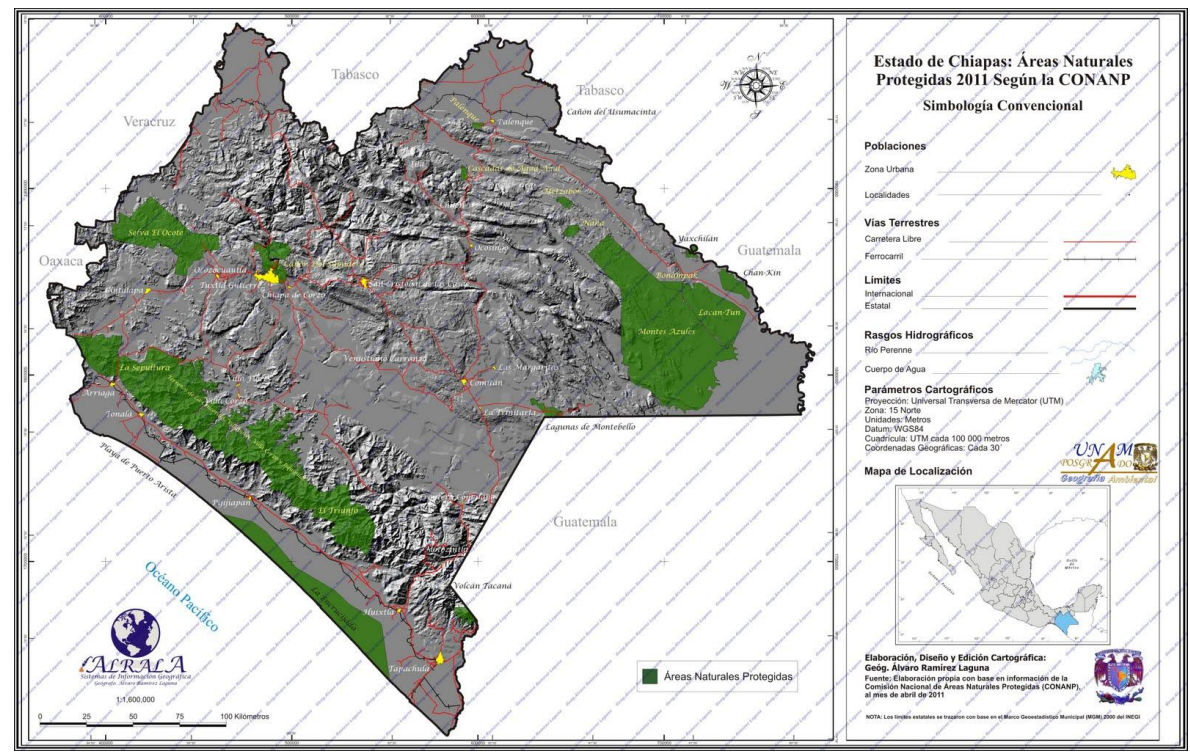

Fuente: Áreas Naturales Protegidas de Chiapas (Ramírez, 2011).

\section{La dinámica económica y la sustentabilidad en La Encrucijada}

La investigación en la Encrucijada considera a las comunidades de Las Garzas, Las Palmas, Barra de Zacapulco, La Lupe, El Conchalito, El Castaño, La Concepción y El Chocohuital, pertenecientes a los municipios de Acapetahua, Mapastepec y Pijijiapan.

La reserva ha sufrido en el transcurso del tiempo, un continuo deterioro, producto de la presión poblacional, ejercida con la explotación de los recursos naturales, que en lo general no ha sido sustentable. Ya que se practican actividades económicas, que causan externalidades en los ecosistemas como la caza, la pesca, el turismo, la ganadería, la agricultura, la industria minera a cielo abierto y la industria azucarera, además de los incendios que afectan a manglares y humedales, aunque hay excepciones en las comunidades de El Castaño y La Concepción, que desde hace varios años han logrado un alto grado de sustentabilidad. 
Los incendios son ocasionados en mayor porcentaje por los cazadores, exterminando importantes extensiones de hectáreas de manglares y humedales, y se presentan con mayor intensidad en el periodo de enero a mayo. Los reportes de la CONANP indican que los mayores riesgos de incendios se dan en los municipios de Acapetahua, Villa Comaltitlán, Huixtla y Mazatán, mientras que los menores se muestran en los municipios de Mapastepec y Pijijiapan (CONANP, 2014).

Se añade a la degradación el cultivo acelerado en los últimos años, por parte de los campesinos, de la palma africana que, de acuerdo con ellos, les genera altos niveles de ingresos económicos, sin embargo, su contrapartida es que están secando los humedales, lo que podría generar la agonía y el colapso de la reserva dentro de pocos años.

Los humedales y manglares han sido dañados por la ampliación de las fronteras de la agricultura y la ganadería; que destruyen los bosques de mangles para el cultivo y usan la madera para cercar los potreros de la ganadería, así como para la construcción de viviendas, de palapas y cabañas destinadas a la actividad turística, siendo bastante crítico en la parte de la Región X del Soconusco.

Otro aspecto que se suma a la degradación de la reserva la Encrucijada, es la continua sedimentación de los humedales y manglares, que se produce por el arrastre de los ríos y arroyos hasta el litoral, provocando que alguna parte de manglares se seque. Lo que altera la ecología de la reserva por el azolve que procede de la Sierra Madre (CONANP, 2014), que se recrudece por el incremento de su deforestación, degradando los suelos y arrastrando los sedimentos hasta la reserva (Villalba, $L a$ Jornada, 2007).

Pero existen otras actividades económicas que operan sin control por parte de las instituciones ambientales y que generan un alto impacto negativo en la reserva, ya que la contaminan con residuos químicos venenosos. Se trata, por un lado, del ingenio azucarero de Huixtla que envenena las cuencas a través de los ríos y después los esteros y lagunas, a falta de una planta de tratamiento de las aguas residuales. No obstante se han gestionado algunas cooperativas de pescadores de la 
zona de influencia en el municipio de Huixtla. Como consecuencia, en 2013 los pescadores del municipio de Huixtla realizaron la denuncia ante la Comisión Nacional del Agua (CONAGUA) y el departamento del Congreso Local de este problema, producido por la fumigación de los cañaverales y el lavado de los molinos del ingenio al inicio de la zafra.

La contaminación que provoca el ingenio azucarero se manifiesta con la muerte de diversas especies, tales como el pijije (especie de pato), pato de agua, nutria y, lo más grave, afecta los ecosistemas en los esteros de Barra de San José en Mazatán, a la población de Villa Comaltitlán, Acapetahua y parte de Huehuetán, lugares en que alrededor de cinco mil personas han sido afectadas al resultar enfermos de cáncer en la sangre y de afecciones en los pulmones (Solís, Diario del Sur, 2013).

Esta contaminación del ingenio, también afecta los cultivos de maíz, mango, piña, pepino y sandía, además de matar a peces como las mojarras, el pez armado, el zambuco, robalo, cocodrilos, nutrias, pululos, garzas, gruyas y plantas acuáticas de la zona (El Sol de México, 2015).

Por otra parte, la reserva también ha sido impactada por la actividad minera de empresas canadienses que explotan los minerales a cielo abierto en la zona de la Sierra Madre de Chiapas, en la que también se utilizan químicos que son arrastrados por los ríos, hasta la reserva y que impactan de manera extraordinaria a los ecosistemas generando ecocidio (Yeé, 2015).

De acuerdo con Zúñiga, en el diario La Jornada (2013), en el estado de Chiapas existen 111 concesiones mineras, siete de ellas están en una zona de 119 mil hectáreas, que limitan con la reserva de La biosfera del Triunfo, de éstas, cinco operan a dos kilómetros de los municipios de Ángel Albino Corzo, Acacoyagua, La Concordia, Mapastepec, Pijijiapan, Siltepec y Villa Corzo.

La Sierra Madre de Chiapas es rica en recursos minerales como el titanio, hierro, plomo y oro, los que generan altos niveles de rentabilidad a las empresas, pero provocan altos costos sociales y ambientales. No obstante, la actividad minera en Chiapas es negada oficialmente, pero 
esta economía es una realidad y una amenaza en la Sierra y en todo el territorio de Chiapas.

La empresa canadiense denominada "Nueva Francia" es un centro minero que se ubica en el municipio de Escuintla, a dos kilómetros del polígono que delimita a la reserva de El Triunfo, poniendo en riesgo tanto a esta reserva como a la reserva de La Encrucijada, así como la vida de las comunidades, tierras agrícolas, aguas y el aire (Bellinghausen, 2014) de las regiones Sierra, Istmo-Costa y Soconusco.

La empresa Blackfire explota el oro a cielo abierto, para lo cual usa cianuro de sodio, plomo, cadmio, arsénico y mercurio; los que además de contaminar el aire también contaminan el agua, ya que penetran hasta los mantos acuíferos.

Los municipios concesionados en la Región del Soconusco e Istmo-Costa para desarrollar la actividad minera con estas características son Acacoyagua, Acapetahua, Cacahoatán, Escuintla, Mapastepec, Pijijiapan y la Concordia (Zúñiga, en chiapasparalelo.com, 2013). Ante este grave problema, grupos ambientalistas como Alianza Sierra Madre y Red Mexicana de Afectados por las Minas han denunciado las políticas de falta de sustentabilidad y efectos nocivos al ambiente, en las que se tienen pocos avances (Bellinghausen, La Jornada, 2014).

\section{Resultados de las políticas de protección ambiental en la Encrucijada}

Si bien la CONANP ha implementado políticas de conservación en la reserva de la Encrucijada, éstas no han logrado detener su degradación, ya que no han sido capaces de integrar, de manera general, a las comunidades y a los actores económicos bajo un modelo de desarrollo sustentable.

Aunque el panorama las políticas ambientales implementadas en la reserva han sido poco eficientes, en el caso de las cooperativas de turismo, Costa verde en la comunidad del Castaño y en la comunidad de La Concepción, se observa un importante nivel de desarrollo sustentable, 
ya que han logrado desde hace muchos años, proteger y conservar los bosques de manglares, humedales y la fauna en la zona núcleo de la Encrucijada, realizando un ecoturismo y una pesca sustentable.

Mientras que, en el resto de las comunidades, acontece una mayor depredación de los recursos naturales, generando desequilibrios de los ecosistemas, incluso se han presentado algunos desacuerdos entre éstas y la CONANP, por la naturaleza de las políticas implementadas y la falta de consciencia ambiental en las comunidades.

Las políticas ambientales que la CONANP ha puesto en operación en La Encrucijada se concretizan en los siguientes programas: en primer lugar, se encuentra la educación ambiental para niños y jóvenes en las escuelas primarias y secundarias, así como pláticas a los socios de las cooperativas pesqueras, con el fin de cambiar la cultura de la población hacia el desarrollo sustentable, sin embargo, esta no ha sido permanente, sino esporádica, por lo que no se ha logrado transformar la cultura ambiental de la población.

En segundo lugar, está la regulación de los permisos otorgados para el uso racional de los recursos en la reserva, como la madera de mangle empleada para la construcción de viviendas, infraestructura de hospedaje y de restaurantes (palapas) para proporcionar servicios turísticos en la zona.

En tercer lugar, se desarrolla el programa de reforestación de mangle rojo (Rhizophora Mangle) en las zonas devastadas, para lo cual se ha organizado e involucrado a la población de las comunidades residentes en la reserva, empleando sus fuerzas de trabajo que generan empleos temporales.

En cuarto lugar, se ha impulsado una actividad pesquera sustentable denominada "pesca responsable" que ha generado un proceso de sensibilización en los pescadores para que practiquen una pesca sustentable, en la que se incluye la vigilancia y monitoreo de las zonas de pesca en épocas de veda y las zonas prohibidas, por parte de los directivos de las cooperativas, como por la CONANP. 
De todos, el principal programa de la CONANP es el de conservación para el desarrollo sustentable (PROCODES) que apoyó a las comunidades de La Encrucijada durante 2013 con 27 proyectos, ejerciendo un presupuesto de 3 millones 19 mil pesos, destinados al ecoturismo, pesca responsable, agricultura, ganadería, restauración ambiental y el programa de empleo temporal.

En la conservación de la Encrucijada, también han participado la Comisión Nacional para el Desarrollo de los Pueblos Indios (CONANP, 2014) y el Fondo Mundial para la Naturaleza (WWF). Se trata de la mayor organización conservacionista independiente del mundo, fundada en abril de 1961 en Suiza, trabaja con 100 países, y la dirección para América Latina se encuentra en Estados Unidos. La misión de esta organización es detener la degradación del medio ambiente natural a nivel mundial, la conservación de la biodiversidad, garantizar la sustentabilidad, promover la reducción de la contaminación y detener el consumo desmedido (www.panda.org).

No obstante, los esfuerzos realizados para transformar la cultura ambiental de la población residente y preservar la reserva, aún sigue prevaleciendo la práctica tradicional, insustentable, por lo que se requiere reforzar la sensibilización ambiental de las comunidades, de los actores económicos y las instituciones gubernamentales encargadas de dirigir la preservación. El intento de establecer en la Encrucijada el paradigma sustentable inicia con la ejecución del proyecto de La Red de Ecoturismo, implementado por la CONANP a partir de 2007, alcanzando sus mayores logros en la comunidad del Castaño, donde se encuentra la cooperativa de turismo Costa Verde.

La Red de Ecoturismo está integrada por las cooperativas de: San Carlos, ubicada en la comunidad de La Palma, que cuenta con un sistema de tratamiento de sus aguas residuales; la cooperativa de El Ballenato ubicada en la Comunidad de La Palma, que construyó un centro turístico y también tiene tratamiento de aguas residuales; la cooperativa Bahía del Mar, ubicada en la comunidad de La Lupe, que produce artículos artesanales; el centro turístico Barra de Zacapulco, también llamado Las Conchitas; y Costa Verde, en la comunidad El Castaño. 
De La Red de Ecoturismo, la cooperativa Costa Verde es la más desarrollada en cuanto a sustentabilidad. La comunidad del Castaño fue funda en 1985 y cuenta con una población de 100 habitantes, perteneciente al municipio de Mapastepec. Su fuente de ingresos y alimentos ha sido primordialmente la pesca, pero se complementa con la actividad ecoturística desde 2007. La comunidad también realiza una pesca sustentable. Otrora, su cooperativa vendía larvas de camarón de sus lagunas y esteros a la granja de camarón de El Fortín, en Pijijiapan. En la actualidad ya no lo hacen porque racionalizan el recurso.

Esta cooperativa construyó su infraestructura turística con ayuda de la gestión de la CONANP, para obtener los recursos financieros procedentes del Banco Mundial y de Francia, aunque estos apoyos dejaron de llegar, actualmente la cooperativa realiza gestiones para conseguir otros con el Banco Mundial, en unión con otras cooperativas turísticas de la región de los Altos de Chiapas.

En cuanto a los recursos financieros procedentes del exterior, La CONANP los ha administrado de acuerdo a su criterio y, lamentablemente, no los ministraba a las comunidades, asunto que fue cuestionado por las comunidades implicadas en el proyecto de desarrollo sustentable, contenido en la Red de Ecoturismo.

Para la vigilancia en la Encrucijada la PROFEPA, que depende de la SEMARNAT, implementa programas, sin embargo, en el caso de la comunidad del Castaño, que estuvo integrada al comité de esta dependencia, se separó debido al cuestionamiento que hicieron al método de vigilancia sugerido por la PROFEPA, pero eso no implicó, que dejaran de vigilar para proteger la biosfera, ya que la comunidad siguió preservando los recursos naturales. 


\section{Cuadro 1. Cooperativas de la Red de Ecoturismo en la reserva de La Encrucijada}

\begin{tabular}{|l|c|c|c|c|c|}
\hline Cooperativas & Actividades & Socios & Localidades & Municipios & $\begin{array}{c}\text { Niveles de } \\
\text { sustentabilidadad }\end{array}$ \\
\hline San Carlos & Turismo & & La Palma & Acapetahua & Nivel medio \\
\hline El Ballenato & Turismo & 16 & La Palma & Acapetahua & Nivel medio \\
\hline Bahía del Mar & $\begin{array}{l}\text { Artesanía y } \\
\text { producción } \\
\text { de pan }\end{array}$ & 10 & La Lupe & Acapetahua & Nivel medio \\
\hline $\begin{array}{l}\text { Barra de } \\
\text { Acapetahua }\end{array}$ & $\begin{array}{l}\text { Transporte } \\
\text { de lanchas }\end{array}$ & & Las Garzas & Acapetahua & Nivel medio \\
\hline $\begin{array}{l}\text { Las Conchitas } \\
\text { o Barra de } \\
\text { Zacapulco }\end{array}$ & Turismo & 110 & $\begin{array}{c}\text { Las } \\
\text { Conchitas }\end{array}$ & Acapetahua & Nivel medio \\
\hline Costa Verde & Turismo & & El Castaño & Mapastepec & Nivel alto \\
\hline
\end{tabular}

Fuente: Elaboración propia.

El alto nivel de conciencia ambiental que ha logrado esta comunidad se puede atribuir, en parte, a que la CONANP capacitó a algunas personas de la comunidad que incluso fueron, por un tiempo, empleados de la institución, convirtiéndose después en los líderes del desarrollo ambiental.

En la zona núcleo de la reserva, también se localiza la Isla de La Concepción, donde existe una comunidad con el mismo nombre. Esta comunidad tiene un destacado mérito en la conservación de la reserva, ya que desde hace sesenta años cuida y conserva la reserva, es decir, antes del decreto de 1995 de La Encrucijada, ya ejecutaba un plan de conservación logrando un importante nivel de sustentabilidad de los ecosistemas, representados por los bosques de manglares, bosques de zapotes, humedales, los recursos pesqueros y la fauna en general.

Según Yeé (2014), aquí los trabajos de conservación institucionalizados fueron inicialmente orientados por el Instituto de Historia Natural, pero cuando la CONANP se hizo cargo de la conservación hubo un estancamiento, ya que la CONANP adoptó un papel asistencialista. Esta 
comunidad no forma parte del proyecto de la Red de Ecoturismo organizada por la CONANP.

Por el momento La Concepción no mantiene buenas relaciones con la CONANP, debido a que considera que sus políticas ambientales implementadas en la reserva, han sido preferenciales porque beneficia con sus proyectos sólo a algunos grupos de otras comunidades. Por eso, los habitantes de la comunidad apelan al voluntarismo y a la autonomía, y no quieren depender de las instituciones ambientalistas gubernamentales.

En la toma de decisiones del Consejo de Asesor Técnico de la Encrucijada, no hay presencia de las comunidades, sólo están algunos representantes comunitarios leales a la CONANP que han creado un grupo de proyectistas, ante los cuales las comunidades deben realizar las gestiones de sus proyectos de desarrollo. Sin embargo, no todos los proyectos son aprobados, y por eso los recursos financieros que llegan a la reserva son selectivos en cuanto a su asignación, además de ser temporales para las comunidades que resultan beneficiadas. Esta es la razón por la que varias comunidades que habitan en la reserva ya no creen, ni se involucran en el proyecto de sustentabilidad que implementa la CONANP.

La globalización económica ha intentado penetrar en el núcleo de la reserva, a través de las propuestas de inversión turística en la construir de infraestructuras hoteleras, pero la comunidad del Castaño las ha rechazado porque aseguran que esto sería el inicio de la acelerada destrucción de La Encrucijada.

Es evidente que, con la modernidad económica, la naturaleza es afectada por los efectos de la contaminación y degradación de los ecosistemas, sobre todo en los espacios de las regiones subdesarrollada, donde se establecen las empresas que explotan los recursos naturales sin que haya un estricto control en la conservación, infringiendo las leyes ambientales a cambio de la obtención de la renta.

Por la problemática ya mencionada en la reserva de la Encrucijada, se sugiere la implementación de las siguientes estrategias que contribu- 
yen a frenar su degradación, al mismo tiempo que permita el desarrollo de las comunidades:

1. Incrementar los niveles de conciencia ambiental, condición necesaria para emprender actividades económicas sustentables, a partir de una pedagogía ambiental por parte de las instituciones ambientales, que incluyan una educación formal e informal, que eduque a las generaciones presentes en las escuelas y fuera de ellas.

2. La difusión de carteles alusivos a su conservación en las comunidades de la reserva.

3. La implementen de spots, en la radio y en la televisión para este mismo fin.

4. Se sensibilice a los actores económicos, por medio de la radio, revistas periódicos, y televisión, para disminuir la degradación de los ecosistemas, al poner en operación sus empresas, en las que utilice métodos y tecnologías amigables con la naturaleza, logrando la armonía entre la economía, la sociedad y la naturaleza.

5. Que los funcionarios del gobierno, en todos los niveles, y en colaboración con la Sociedad Civil, apliquen las leyes ambientales a todas las personas y empresas de manera indiscriminada, evitando las acciones de corrupción.

6. Que haya por medio del gobierno el apoyo en la implementación de proyectos de desarrollo económico alternativos en las comunidades para diseccionar la presión sobre la reserva, aunque se reconoce que estamos en un modelo económico que le ha quitado al gobierno la responsabilidad de ayudar a los pueblos a superar sus múltiples problemas económicos, sociales y ambientales (como ocurría en el modelo Keynesiano), no solo en México, sino en casi todos los países de América Latina. No obstante, debe haber apoyos ya que la sociedad mexicana cada vez está más pobre.

El panorama de la crisis ambiental del mundo podría cambiar siempre que haya consciencia del problema, la voluntad y consenso por parte de los actores sociales, económicos y políticos. Lo que permitiría cambiar 
los paradigmas económico, político, legal y filosófico, que garanticen la conservar de la naturaleza y de la humanidad, construyendo modelos de desarrollo sustentable y el mejoramiento de las condiciones de vida humana, aunque parezca utópico.

\section{Conclusiones}

Los problemas ambientales actuales han sido resultado de la crisis civilizatoria, tienen su origen en la práctica de una economía que depreda y sobreexplota los recursos naturales, en el crecimiento demográfico mundial, en la falta de conciencia ambiental de la población mundial en general.

Los problemas de degradación comienzan a manifestarse desde la primera Revolución Industrial del siglo XVIII, y se ha acrecentado hasta convertirse en un asunto casi irresoluble. La crisis ambiental se manifiesta en el desequilibrio de los ecosistemas, en el agotamiento de los recursos naturales, como los recurso pesqueros, los forestales, la fauna y el agua dulce, para los cultivos y el consumo humano; así como la contaminación del suelo, del aire, de los ríos, mares y océanos; el calentamiento global, y la reducción de la capa de ozono.

Los antecedentes demuestran que no es sino hasta la década de los cincuenta del siglo XX cuando los investigadores comienzan a preocuparse, y en la década de los sesentas y setentas del mismo siglo aparece la disyuntiva de si continuar con el modelo de desarrollo occidental o cambiarlo por otro.

El problema ambiental, generó que la ONU impulsara su primera cumbre en Estocolmo, Suecia, en 1972. Como resultado de ésta se retomó el derecho que todos tenemos a un ambiente sano y se adoptó el nuevo modelo de Ecodesarrollo como alternativa. Para la década de los ochentas, todas las teorías económicas y sus estrategias de desarrollo entraron en crisis, sobre todo en los países subdesarrollados. Por lo que se plantearon nuevos modelos de desarrollo sustentable. 
La ONU organizó otras cumbres internacionales, como la de 1992, en Río de Janeiro, Brasil, y, en 2000, en Johannesburgo, Sudáfrica, pero desafortunadamente no se han logrado los resultados requeridos, ya que la tierra sigue agonizando. Dentro de las propuestas de solución, la ONU implementó un programa de Educación Ambiental formal y no formal, en la cual participó la UNESCO, aunque tampoco ha podido transformar la cultura antropocéntrica de la mayor parte de la población mundial.

También se ha intentado resolver el problema ambiental a partir de transformar las practicas económicas, en cuanto a cambiar la racionalidad económica por la racionalidad ambiental. Por lo anterior, se ha implementado un nuevo paradigma epistemológico que proponga y dé solución a los problemas ambientales, como la Economía Ecológica o Economía Verde que considera a los ecosistemas como unidades de análisis, a los que no se deben ver como valores de cambio, sino como valores de uso y bienes públicos.

En México, las políticas ambientales para la conservación de la biodiversidad comienzan en 1876 con la protección del Desierto de los Leones. Para los setentas, esta política de protección es contradictoria, ya que se fomenta el poblamiento de las zonas boscosas y selváticas del país (como la Selva Lacandona en Chiapas) y se permite la deforestación para practicar actividades agrícolas y ganaderas.

En cuanto al estado de Chiapas, se encontraron algunas áreas protegidas de la biodiversidad dentro de las cuales está La Encrucijada, en la que no se observa uniformidad en cuanto a su conservación. Ya que hay zonas que se mantienen casi inalteradas, mientras que otras presentan un importante deterioro, casi están exterminadas, como el caso de algunas zonas de los municipios de Mazatán, Huixtla, Villa de Comaltitlán y Pijijiapan.

A pesar de las políticas ambientales puestas en marcha, no se ha logrado detener la degradación de la reserva. Contribuyen a este problema, la falta de sustentabilidad en las actividades económicas que se practican, y a la ausencia de un nivel de consciencia ambiental en los actores sociales tales como empresarios, sociedad civil y gobierno, que 
no asume su papel de garantizar la conservación de los ecosistemas, sin flexionar por presiones.

En primer lugar, en la Encrucijada se desarrollan actividades agrícolas y ganaderas, de forma tradicional y extensiva, para lo cual deforestan manglares. En segundo lugar, la reserva es impactada por la elevada contaminación que generan la industria azucarera ubicada en el municipio de Huixtla; la minera canadiense, que se desarrolla a cielo abierto, extrayendo oro, titanio, entre otros metales más viles, y que opera en distintos lugares de la Sierra Madre de Chiapas, sin embargo, sus desechos químicos venenosos, están impactando en la reserva, destruyendo los ecosistemas y ocasionando enfermedades terminales en la población humana. Se suman la degradación, los incendios que devastan grandes extensiones de la reserva, provocados por los cazadores clandestinos que incendian humedales y manglares, y con éstos, la muerte de especies de mamíferos y reptiles.

El inicio de las políticas ambientales de conservación de la reserva se da por parte del IHN desde 1991, fortaleciéndose con el Decreto de Reserva de La Encrucijada en 1995 y haciéndose cargo de su preservación la CONANP, que deriva de la SEMARNAT.

Sin embargo, no se ha logrado detener de manera significativa las externalidades negativas de las actividades económicas, y tampoco se ha logrado transformar uniformemente la cultura ambiental de la población en cuanto a la sustentabilidad. Las únicas comunidades que han conseguido un alto grado de conciencia ambiental y de desarrollo sustentable son El Castaño y La Concepción.

Si la sustentabilidad no se logra homogeneizar en La Encrucijada, ésta se verá agonizar en el transcurso del tiempo, como lo están haciendo otras reservas de la biosfera en el mundo, tales como La selva Lacandona de Chiapas, la selva de Las Amazonas, entre otras regiones de reserva. Por consiguiente, de nada habrá servido la abundante investigación, las diversas teorías y discursos intelectuales, de los gobiernos nacionales y de los organismos internacionales como la ONU y la UNESCO. 


\section{Bibliografía}

Börzel, T., "¿Qué tienen de especial los Policy Networks? Explorando el concepto y su utilidad para el estudio de la gobernación europea", European Integration Online Papers, vol. 1, Italia, 1997. En http://revista redes rediris. es/webredes/textos/policynet.doc.

Borrego, P. N., "Políticas Públicas de Protección al Medio Ambiente en Norteamérica", Revista mexicana de Estudios Canadienses (nueva época), núm. 011, México, 2006, pp. 163-192.

CONANP, Programa Nacional de Áreas Naturales Protegidas, 20072012, Ciudad de México, 2012. , La encrucijada, Ciudad de México, 2014.

Contreras, D. Wilfrido, Planeación Ambiental Local. Planeación en México. Región y Ambiente, Facultad de Planeación Urbana y Regional de la UAEM, Estado de México, 2002.

González, Adrián, Acaban con los manglares en Puerto Chiapas ante la indiferencia de SEMARNAT, Ecosur, México, 2012.

Gutiérrez, Nájera Raquel, "El desarrollo sustentable: un camino a seguir", Espiral, vol. 11, núm. 5, Universidad de Guadalajara, 1996, pp. 197-227. En http://www.redalyc.org/artículo. Oaid=13820509.

Hufty, M., Una propuesta para concretizar el concepto de gobernanza: El Marco Analítico de la Gobernanza, 2008, p. 17.

Landa, Rosalva, Brenda Àvila Flores y Mario Hernández, Cambio Climático y desarrollo sustentable para América Latina y el Caribe. Conocer para comunicar. BRITISH COUNCIL, México, 2010. En http://icons.mysitemyway.com/

Leff, E., Cambio climático, energía y desarrollo sustentable. En Los problemas del conocimiento y la perspectiva ambiental del desarrollo, Siglo XXI, México, 2000.

, "La Geopolítica de la Biodiversidad y el Desarrollo sustentable: Economización del mundo, racionalidad ambiental y rea- 
propiación Social de la naturaleza", Seminario Internacional REG GEN: Alternativas Globalizadas, UNESCO, Rio de Janeiro Brasil, 8 al 13 de octubre de 2005. En: www: http://bibliotecavirtual.CLACSO.ORG.ar/libro

, Discursos Sustentables, Ambiente y democracia, Siglo XXI, México, 2008, p. 272.

Martínez, C. R., "Importancia de la educación ambiental ante la problemática actual", Revista Electrónica Educare, vol. XIV, núm. 1, enero-junio, 2010, pp. 97-111.

Nowlin, M. "Theories of the Policy Process", State of the Research and Emerging Trends The Policy Studies Journal, núm. 39, vol. 1, 2011, pp. 41-60.

Ostrom, E., "Institutional Rational Choice: An Assessment of the Institutional analysis and development framework", en P. Sabatier (ed.), Teories of the Policy Process, United States Westview Press, 2007. pp. 21-64.

Roth, André-Noel, Políticas Públicas: Formulación, implementación y evaluación. Ediciones Aurora, Colombia, 2002, pp. 134.

SEMARNAT. Programa anual de trabajo, 2012.

Toro, S. F. J., El desarrollo sustentable: un concepto de interés para la Geografía, Departamento de Análisis Geográfico Regional y Geografía Física de la Universidad de Granada-Cuadernos Geográficos, 2007, pp. 149-181.

Torres, Melo, Jaime y Santander Jairo, Introducción a las Políticas Públicas: Conceptos y herramientas desde la relación entre Estado y Ciudadanía, Instituto de Estudios del Ministerio Público, Procuraduría General de la Nación, Colombia, 2013, p. 172. En: http://Iemp.procuraduria.gov.CO.

\section{Bibliografía digital}

Bellinghausen, "La minería en Chiapas", La Jornada, jueves 3 de abril, 2014. 
"Contaminación proveniente del ingenio azucarero de Huixtla- afecta sistema lagunero. Tapachula, Chiapas", Diario El Orbe, martes 31 de octubre, 2017. En: htts: elorbe.com/portada.2017/04/26/ contaminación proveniente-el ingenio-azucarero-afecta-el sistema lagunario

Villalba, Sánches Rodolfo, "Peligro de extinción de la reserva de La Encrucijada Chiapas", La Jornada, 18 de agosto, 2007.

Zúñiga, "Concesiones mineras en Chiapas", La Jornada, 29 de septiembre, 2013

Solís Cesar, "Ingenio azucarero afecta sistema lagunario de la costa de Chiapas", Diario del Sur. En: https:/www.inforural.com.mx

http://www. Conanp.gob. mx/comunicado de prensa.

http://www. Chiapasparalelo.com/opinión/2014/03/el porqué del fracaso de la conservación de la Encrucijada.

http://www. México desconocido.com.mx/reserva de La Encrucijada.

[Consultado: 6 de marzo de 2015]

http://www. Noticiasnet.mx/contaminación, ingenio azucarero de Huixtla, Chiapas, jueves 30 de abril, 2015.

http://www..conanp.gob.mx/acciones/procodes.php/ La Encrucijada. 28 de junio de 2014.

http://www.chiapasparalelo.com/2013/precisiones/de la conanp sobre la Encrucijada. 7 de julio de 2013.

http://www.chiapasparalelo.conservacion y desarrollo sustentable en la Encrucijada, 2014. 5 de mayo de 2014.

http://www.mexicodesconocido.com.mx/reserva de la Encrucijada. 14 de mayo de 2014.

http://www.panda.org. 25 de octubre de 2015. 
